\title{
Clinical Experience of an Effective Treatment of Intractable Chronic Venous Ulcer on the Lower Leg Using Dual-Frequency Ultrasound
}

\author{
Yean Su Choi \\ Seung Min Nam \\ Eun Soo Park
}

Department of Plastic \& Reconstructive Surgery, Soonchunhyang Bucheon University Hospital, Bucheon, Korea
Received June 20, 2017

Accepted June 21, 2017

\footnotetext{
Correspondence

Eun Soo Park

Department of Plastic \& Reconstructive Surgery, Soonchunhyang Bucheon University Hospital, 170, Jomaru-ro, Wonmi-gu, Bucheon 14584, Korea

Tel.: +82-32-621-5319

Fax: $+82-32-621-5662$

E-mail: peunsoodschmc.ac.kr

(C) Korean Society for Laser Medicine and Surgery

(@) This is an open access article distributed under the terms of the Creative Commons Attribution NonCommercial License (http://creativecommons.org/ licenses/by-nc/4.0) which permits unrestricted noncommercial use, distribution, and reproduction in any medium, provided the original work is properly cited.
}

We report a case of intractable venous ulcer that was effectively treated by using local dynamical micro-massage (LDM ${ }^{\circledR}$-MED; Wellcomet, Karlsruhe, Germany). A 43-year-old male visited a hospital with chief complaint of painful wound on his left lower leg. The patient crashed his leg against a bicycle, and the wound has not recovered for the past 6 months. There were many features indicating venous ulcer, such as edema, hyperpigmentation, eczematous changes with erythema, and obesity. Despite our attempt of surgical intervention, involving debridement and split thickness skin graft, the raw surface had not cured completely. We applied LDM to the wound to activate the healing process by modulating the activity of matrix metalloproteinases and heat shock proteins. The result was impressive. After 21 LDM treatments in a 5 week period, the wound was totally re-epithelized, and the patient no longer felt pain.

\section{Key words}

Venous ulcer; Compression bandage; Ultrasound 


\section{MEDICAL}

\section{INTRODUCTION}

There are numerous types of chronic wounds; the three most common are pressure sore, diabetic gangrene, and venous ulcer. The $84 \%$ of venous ulcers are located in the lower legs, especially around the ankle.' Although these wounds have different pathophysiology from one another, they have similar attributes of cure: Local inflammation, growth of granulation tissue, re-epithelization of wounds, and remodeling of extracellular matrix. A set of these courses has a close relationship with the balanced movement among collagen circulation between production (regulated by mesenchymal cells) and decomposition (controlled by different enzymes from the matrix metalloproteinases [MMPs]) in connective tissue. Recently two theories respectively based on over-expression of MMPs in the wound bed and under-expression of heat shock proteins (HSPs) were proposed and considered as the most accurate ideas which can explain the pathophysiology of the chronic wounds. ${ }^{2,3}$ Until now, the most well-known method to treat venous ulcers was high compression bandaging which has definite effect to reduce the production of MMPs. ${ }^{4}$ Besides, the application of ultrasound to chronic wounds also showed significant improvement of wound state in many reports. ${ }^{5}$ So, the ultrasound, especially in the form of high frequency such as $10 \mathrm{MHz}$, is focused on and is considered for the ideal treatment of chronic wounds involving venous ulcer.

\section{CASE REPORT}

\section{Patient and method}

A 43-year-old male complained of painful raw surface on his left lower leg which was triggered by direct trauma with bicycle. Because the wound was not healed naturally until 6 months passed, he was admitted into a hospital and had operation. When the wound bed became clear after we performed debridement at first, skin graft was performed twice about 1 month apart to cover the $7 \times 6$ $\mathrm{cm}$ sized raw surface. Due to continuous discharge and chronic inflammation on wound, skin graft didn't show successful result. Therefore, instead of further surgical procedure, non-invasive treatments such as local dynamical micro-massage (LDM ${ }^{\circledR}$-MED; Wellcomet, Karlsruhe, Germanyl and high compression bandaging were considered as an alternative.

We have conducted LDM treatment 6 times a week during 2 weeks of hospitalization. After discharge, the patient had visited hospital and received LDM treatment 3 times a week during 3 weeks. Each treatment lasted 6 minutes along the wound site, with parameters as follows: LDM $10 \mathrm{MHz}$ for 3 mins, $3 / 10 \mathrm{MHz}$ for 3 mins, the intensity of $0.5 \mathrm{~W} / \mathrm{cm}^{2}$. During the treatment, wound site and surrounding tissue in $3 \mathrm{~mm}$ distance were treated equally. We used ultrasonic gel as coupling medium.
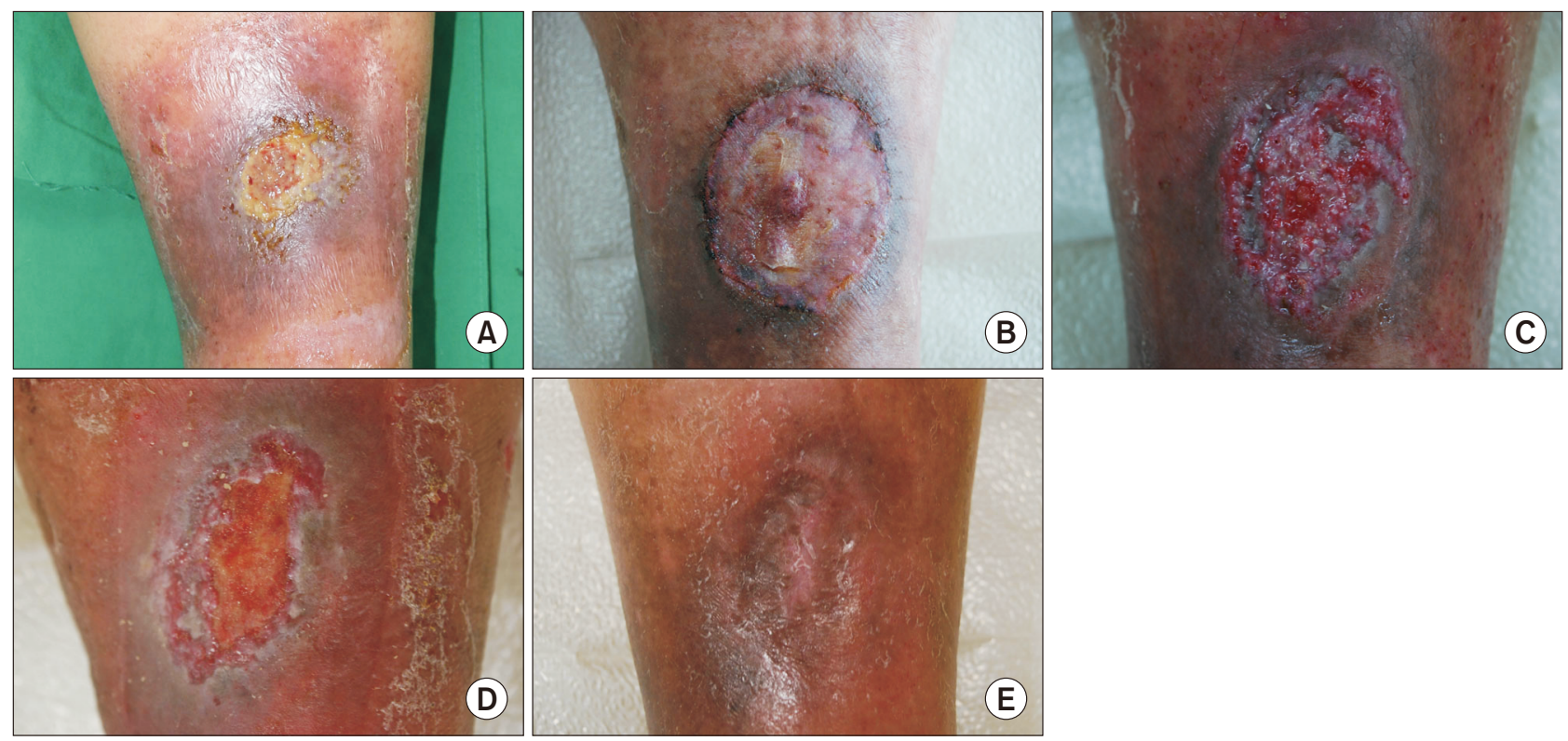

Fig. 1. 43-year-old male with chronic venous ulcer on left lower leg after direct trauma with bicycle. (A) Before treatment. (B) After second skin graft operation. (C) Graft was not taken and venous ulcer recurred. (D) After 12 sessions of LDM. (E) After 21 sessions of LDM. 


\section{RESULT}

During the treatment using LDM, the patient has shown the dramatic wound granulation. Wound treated until the end of the treatment was completely re-epithelized. After the termination of the treatment, we conducted followup research, which showed no pain or side-effects were submitted. The patient complained pain as much as 7 or higher scales according to NRS (numerical rating scale) before treatment. At the end of the treatment there was dramatic decrease in pain as 0 or 1 scale according to NRS.

\section{DISCUSSION}

Venous ulcer is one of the most difficult wounds to treat in modern medical science. This wound is usually intractable to both medical and surgical methods and recurs well so it tends to prolonged for a long time. Newly occurred and small sized venous ulcers on lower legs are often recovered spontaneously and also responding well to treatments, such as high compressive bandaging. Unlike these good prognostic wounds, old and big venous ulcers on lower legs resist therapy and not be improved well in spite of enough time to treat. With extended duration of treatment, patient's life quality declines and the medical expenses are overcharged. Therefore we should develop new method which can not only shorten the therapeutic period but achieve a better effect.

Recently, many studies focus on the effectiveness and safety of the treatment of venous ulcers on lower legs by using LDM therapy. In 2011, Kruglikov and Kruglikova' reported 10 cases of patients with chronic venous ulcer on lower legs treated with LDM 8-25 times. All patients showed remarkable clinical outcomes, especially chronic wounds became fully epithelized and pain was released at least 3 grades according to NRS scale.

In the dual-frequency LDM wave, the frequency is not constant but oscillates between two frequencies $(3$ and 10 $\mathrm{MHz}$ in this case). Changing frequencies every $5 \mathrm{~ms}$ (200 times per second) is effective to decrease MMPs and to stimulate HSPs so it helps wound healing process. Actually in our case, we can observe the wound was recovered completely at the end of treatment. Patient accepted LDM treatment well because there was no pain or side effect during whole treatment period.

Because high compressive bandaging was not able to achieve competent healing, bandaging was stopped during LDM therapy in most of previous studies. We always maintained bandaging except performing LDM therapy in this case and clinical result was excellent. Although our result is based on only one case, we expect more patients with venous ulcer will be cared successfully with our LDM protocol. In addition, it would be interesting to study another topics such as the role of high compressive bandaging during LDM protocol and how often the treatment should be performed to achieve the best result. Future studies of large scale dealing with these issues will build up a well-organized formula of treatment with reliable statistical results.

\section{REFERENCES}

1. Kruglikov I, Kruglikova E. Dual treatment strategy by venous ulcers: pilot study to dual-frequency ultrasound application. J Cosmet Dermatol Sci Appl 2011;1:157-63.

2. Cook H, Davies KJ, Harding KG, Thomas DW. Defective extracellular matrix reorganization by chronic wound fibroblasts is associated with alterations in TIMP-1, TIMP-2, and MMP-2 activity. J Invest Dermatol 2000;115:225-33.

3. McMurtry AL, Cho K, Young LJ, Nelson CF, Greenhalgh DG. Expression of HSP70 in healing wounds of diabetic and nondiabetic mice. J Surg Res 1999;86:36-41.

4. Renò F, Grazianetti P, Stella M, Magliacani G, Pezzuto C, Cannas M. Release and activation of matrix metalloproteinase-9 during in vitro mechanical compression in hypertrophic scars. Arch Dermatol 2002;138:475-8.

5. Roche C, West J. A controlled trial investigating the effect of ultrasound on venous ulcers referred from general practitioners. Physiotherapy 1984;70:475-7. 